\title{
Parechovirus como agente etiológico de meningitis y/o sepsis viral en lactantes
}

\author{
Valentina Gutiérrez, Constanza Martínez-Valdebenito, Luisa Montecinos, \\ Romina Alarcón, Constanza Gárate y Marcela Ferrés
}

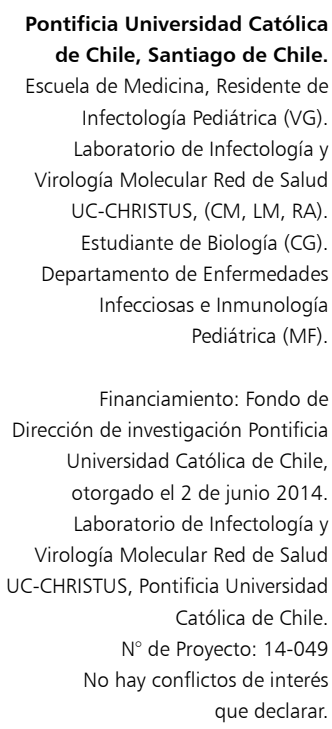

Pontificia Universidad Católica de Chile, Santiago de Chile. Escuela de Medicina, Residente de Infectología Pediátrica (VG) Laboratorio de Infectología y Virología Molecular Red de Salud UC-CHRISTUS, (CM, LM, RA). Estudiante de Biología (CG) Departamento de Enfermedades Infecciosas e Inmunología Pediátrica (MF)

Financiamiento: Fondo de Dirección de investigación Pontificia Universidad Católica de Chile, otorgado el 2 de junio 2014 Laboratorio de Infectología y Virología Molecular Red de Salud UC-CHRISTUS, Pontificia Universidad Católica de Chile.

$N^{\circ}$ de Proyecto: 14-049 No hay conflictos de interés que declarar.

Recibido: 5 de enero de 2016 Aceptado: 17 de junio de 2016

Correspondencia a: Marcela Ferrés Garrido mferres@med.puc.cl

\section{Introducción}

E 1 síndrome febril agudo en niños bajo dos años (lactantes) es un motivo de consulta pediátrica frecuente. Se estima que $60 \%$ de las consultas en el Servicio de Urgencia de niños bajo 36 meses de edad corresponden a este síntoma; las causas bacterianas representan entre 10 y $25 \%$, en tanto las virales 72 a $76 \%{ }^{1}$. Entre estas últimas, las sepsis virales y el compromiso del sistema nervioso central (SNC) representan un mayor desafío diagnóstico y terapéutico. Las infecciones virales sistémicas en este grupo etario pueden presentarse con síntomas inespecíficos, rechazo alimentario, fiebre, irritabilidad, y excepcionalmente, muerte ${ }^{2}$.

Es así como, ante un lactante con síndrome febril agudo, la primera disyuntiva es el diagnóstico diferencial entre una infección bacteriana y una infección viral sistémica. Este hecho cobra especial relevancia al momento de hospitalizar, decidir el uso de terapias antimicrobianas empíricas y, posteriormente, la suspensión de éstas cuando la evolución no ha sido compatible con una infección bacteriana. La estadía hospitalaria, en espera de resultados microbiológicos, contribuye al uso injustificado de antimicrobianos y aumenta el riesgo de infecciones asociadas a la atención de salud (IAAS), descritas en 4,5 por 100 admisiones hospitalarias ${ }^{3,4}$. Por ello, acceder a un diagnóstico virológico amplio y oportuno puede influir en reducir la adquisición de IAAS y permitiría el uso de antivirales específicos, de haberlos disponibles 5 .

El rápido desarrollo de la virología clínica y molecular ha permitido contar con un espectro etiológico creciente de agentes virales. Es así como se descubrió un nuevo agente, parechovirus humano ( $\mathrm{HPeV})$, inicialmente descrito dentro del género de Enterovirus (EV) como echovirus 22 y 23 en un brote de diarrea en niños en Estados Unidos de América (E.U.A.) en 1952 ${ }^{6}$. Posteriormente, su estudio molecular evidenció diferencias en el genoma, proteínas y propiedades biológicas con respecto a los EV, siendo renombrados en 1999 como HPeV tipo 1 y $2^{7,8}$. Más tarde, $\mathrm{HPeV}$ ha sido descrito como agente causal de sepsis viral y meningoencefalitis ${ }^{8-10}$.

Los $\mathrm{HPeV}$ pertenecen a la familia Picornaviridae, son virus pequeños, desnudos, con ARN de hebra simple, de polaridad positiva. Su genoma contiene 7.300 pares de bases (pb) que codifican por una poliproteína única, flanqueada en los extremos 5' a 3' por regiones no traducidas (UTR, por sus siglas en inglés). El genoma de 
$\mathrm{HPeV}$ es traducido a una poliproteína que es procesada proteolíticamente, dando como resultado tres proteínas virales estructurales (VP0, VP3 y VP1) y siete proteínas no estructurales (2A-2C y 3A-3D). Para el diagnóstico de $\mathrm{HPeV}$ se amplifica una región conservada del extremo 5'UTR de $194 \mathrm{pb}$, en tanto para la genotipificación se secuencia una región de unión entre VP3 y VP1 de 304 $\mathrm{pb}^{11,12}$.

El patrón epidemiológico, al igual que EV, es estacional, donde los casos se concentran a fines de verano y otoño ${ }^{10}$. Su período de incubación es desconocido, en tanto, la transmisión parece ocurrir a través de la vía fecal oral y/o vía respiratoria ${ }^{13}$. $\mathrm{HPeV}$ se excreta durante una a tres semanas en secreciones respiratorias y hasta meses en deposiciones, con promedio de 51 días ${ }^{14,15}$.

La población preferentemente afectada corresponde a niños de tres años o menos, siendo descrito con mayor frecuencia bajo un año ${ }^{16}$. Dentro de su espectro clínico se han referido síntomas gastrointestinales, respiratorios, exantema, sepsis viral neonatal, meningoencefalitis, hepatitis, miocarditis, convulsiones y muerte ${ }^{17-19}$.

El diagnóstico de sepsis o meningitis por HPeV se realiza mediante transcripción reversa y reacción de polimerasa en cadena (RPC-TR), que es el método de elección por su alta especificidad y sensibilidad ${ }^{11,20,21}$. Las muestras utilizadas para el diagnóstico y detección del virus alcanzan diferentes sensibilidades siendo mayor en deposiciones $(95,1 \%)$, líquido cefalorraquídeo (LCR) $(84,1 \%)$, sangre $(79,3 \%)$ y menor en orina $(56,8 \%)^{22}$. Su especificidad es de $100 \%$ en la identificación de los parechovirus hasta ahora descubiertos ${ }^{11,22}$.

Se conocen 16 genotipos, y recientemente se ha descrito un nuevo genotipo $17^{23}$, siendo más frecuentes en infecciones pediátricas $\operatorname{los} \mathrm{HPeV} 1,2$ y 3; el tipo 3 es asociado con mayor frecuencia a enfermedad más grave $^{24-26}$.

La incorporación de este nuevo agente en la búsqueda de etiologías virales ante la sospecha de una enfermedad viral grave, incrementaría los porcentajes actuales de pesquisa global de $40 \% 5$ a $43-48 \%$, dado que su prevalencia descrita en estudios previos varía entre 3 y $8 \%$. Las cifras más bajas fueron detectadas en muestras de LCR de pacientes bajo 18 años, mientras que las cifras mayores se encontraron en muestras de LCR y deposiciones en lactantes, predominando la positividad en lactantes bajo 3 meses $^{15,17,19,27}$.

El propósito de este estudio fue estimar la frecuencia de $\mathrm{HPeV}$ en lactantes con sospecha de infección viral del SNC o sepsis viral, durante el período de octubre 2013 a marzo 2015. Junto a esto, caracterizar la presentación clínica de los pacientes infectados, identificar los genotipos circulantes y contribuir al conocimiento clínico epidemiológico del espectro de infecciones virales graves en lactantes.

\section{Materiales y Métodos}

\section{Estudio descriptivo}

\section{Poblaciones de estudio}

Lactantes, en quienes se solicitó estudio etiológico viral ante la sospecha de infección viral del SNC y/o sepsis viral. Estos pacientes se incorporaron al estudio en dos grupos:

Pacientes con muestras de colección del Laboratorio de Infectología y Virología Molecular. Muestras de LCR y/o plasma enviadas para estudio viral al Laboratorio de Virología Molecular. Estas muestras provenían del Hospital Clínico UC y de los otros Centros de la Red de Salud UC-CHRISTUS. Para efectos del estudio, se incluyeron muestras de deposiciones e hisopado nasofaríngeo (HNF), si se encontraban disponibles. Cada muestra fue originalmente enviada y procesada por técnicas de reacción de polimerasa en cadena (RPC) y RPC-TR en tiempo real para uno o más de los siguientes virus: enterovirus (EV), virus herpes simplex 1 y 2 (VHS 1 y VHS 2), virus varicela-zoster (VVZ), virus herpes hominis 6 (HHV6), adenovirus (ADV), parvovirus B19 (PvB19). Las muestras de deposiciones utilizadas fueron estudiadas previamente a través de técnica de RPC para seis virus entéricos (ADV, norovirus $\mathrm{G} 1$ y G2, astrovirus, rotavirus y $\mathrm{EV}$ ) y en los HNF se estudió la presencia de 15 virus respiratorios (virus respiratorio sincicial $\mathrm{A}$ y $\mathrm{B}$, influenza A y B, parainfluenza 1, 2, 3 y 4, metapneumovirus, coronavirus oc43, coronavirus 229e/nl63, ADV, EV, rinovirus y bocavirus). Se excluyeron muestras de LCR hemorrágico o muestras escasas. Todas las muestras fueron apropiadamente almacenadas a $-80^{\circ} \mathrm{C}$ hasta $\mathrm{su}$ procesamiento.

Las muestras de estos pacientes fueron recibidas en el laboratorio para estudio virológico entre octubre del año 2013 y enero del año 2015.

Pacientes hospitalizados en establecimientos de la Red Salud UC-CHRISTUS: Lactantes con sospecha diagnóstica de sepsis y/o meningoencefalitis evaluados clínicamente por los investigadores.

Criterios de inclusión: Fiebre (temperatura $\geq 38^{\circ}$ rectal), y al menos dos de los siguientes síntomas: irritabilidad, somnolencia, fotofobia, marcado rechazo alimentario, vómitos, exantema y/o al menos un signo de infección sistémica como hipotermia, hipovolemia o shock.

Criterios de exclusión: Pacientes con otros diagnósticos, que al momento de la toma de muestras explicara la sintomatología y, rechazo de consentimiento informado.

Estos pacientes fueron ingresados al estudio entre septiembre 2014 y marzo 2015. 


\section{Tamaño de muestra}

Se calculó un tamaño de muestra mínimo de 74 pacientes, con frecuencia de hallazgo de parechovirus estimada en $3 \%$ y se usó una potencia de $80 \%$ y error de $3 \%$ (28).

\section{RPC-TR diagnóstica}

Para la amplificación del segmento de 5'UTR se utilizó el protocolo descrito por W. Allan Nix y cols (11). Brevemente, se realizó transcripción reversa; $5 \eta \mathrm{g}$ de hexámeros, 5U de MMLV (transcriptasa reversa) y $0,2 \mathrm{mM} \mathrm{dNTP}$. La reacción de transcripción reversa se realizó a $37^{\circ} \mathrm{C}$ durante $40 \mathrm{~min}$. La amplificación de cADN se realizó mediante RPC en tiempo real. Tanto sonda como partidores se utilizaron a una concentración de 0,2 mM; para amplificación se utilizó ADN master plus (ROCHE®). El programa de RPC fue $95^{\circ} \mathrm{C} 5 \mathrm{~min}$ seguido de 35 ciclos a $95^{\circ} \mathrm{C} 15$ segundos, $60^{\circ} \mathrm{C} 1$ minuto.

Los controles positivos de ARN fueron donados por el Dr. Steven Oberste del CDC (Centro de Control y Prevención de Enfermedades, E.U.A.).

La infección fue confirmada al detectar ARN de parechovirus en uno de los siguientes fluidos: LCR y plasma, y de apoyo al diagnóstico: deposiciones o HNF.

\section{Caracterización genotípica}

Para la caracterización genética e identificación de los tipos de parechovirus de las muestras positivas se compararon las secuencias ubicadas en la región VP3/ VP1 (304 pb). A través de la amplificación mediante RPC, secuenciación y el uso de herramientas bioinformáticas se determinó el tipo de parechovirus ${ }^{12}$. Esta región ha sido previamente identificada como altamente sensible y específica para la tipificación de todos los tipos de parechovirus $^{11,29}$.

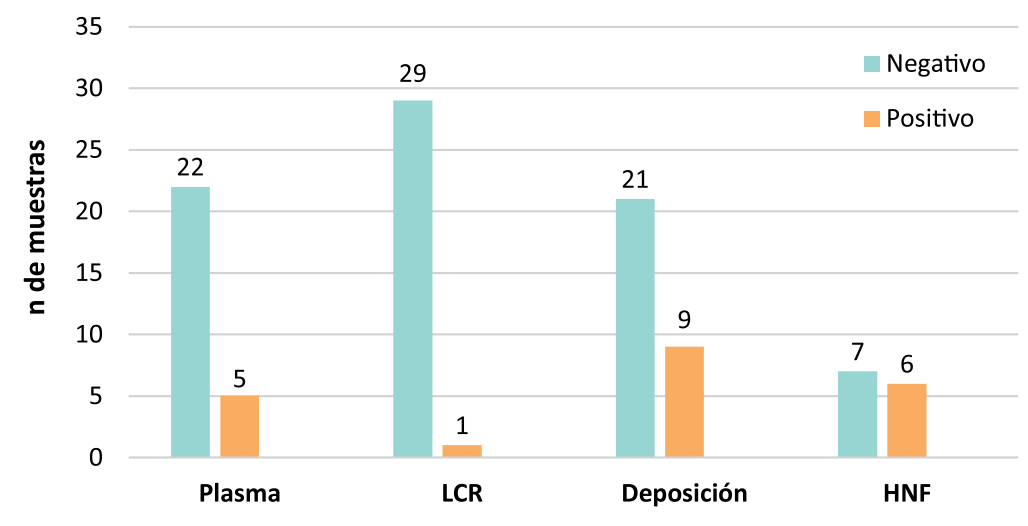

Figura 1. Frecuencia de $\mathrm{HPeV}(+)$ en 100 muestras analizadas. HNF: hisopado naso-faríngeo.

\section{Análisis estadístico}

Se utilizó test de $\chi^{2}$ para estimar diferencias en las poblaciones estudiadas y test de Fisher si los sujetos a comparar eran grupos menores a cinco. Se utilizó el programa Statistical Package for the Social Sciences (SPSS) versión 21.

\section{Aspectos éticos}

El estudio contó con aprobación del Comité Ético Científico de la Escuela de Medicina de la Pontificia Universidad Católica de Chile No 14-049. Con fecha 6 de mayo 2014, se aprobó dispensa de consentimiento para uso de muestras de colección respetando el anonimato de los pacientes. Con fecha 5 de agosto 2014, se aprobó proyecto y consentimiento informado para incorporar pacientes hospitalizados y 19 de marzo 2015 aprobación para el uso de material fotográfico.

\section{Resultados}

\section{Descripción de la muestra}

Se recolectó un total de 100 muestras para estudio, pertenecientes a un total de 59 pacientes (46 del grupo "muestras de colección” y 13 del grupo "hospitalizados"). La distribución de edad fue entre 1 día y 1 año 11 meses, con un promedio de 4 meses. Del total de pacientes analizados, una mayor proporción de pacientes eran hombres $(55,9 \%)$, dos tercios se encontraban hospitalizados al momento de la toma de muestra y un tercio del total de pacientes fue ingresado a la Unidad de Paciente Crítico Pediátrico (UPCP) por su gravedad.

\section{Determinación virológica}

De las 100 muestras analizadas, 21 fueron positivas para $\mathrm{HPeV}$. Las 21 muestras positivas, provenían de 11 pacientes, 8,7\% (4/46) del primer grupo y 53,8\% (7/13) del segundo grupo de estudio, dando una estimación global de hallazgo de HPeV de 18,6\% (11/59). En la Figura 1 se observa la distribución de éstas, según tipo de fluido biológico, destacando la muestra de deposiciones por su positividad.

La genotipificación fue exitosa en 6 de los 11 pacientes, siendo el genotipo HPeV tipo 3 el único encontrado en todas las muestras.

Previo al diagnóstico de HPeV, 17/59 (28,8\%) del total de pacientes tenían un diagnóstico etiológico viral, siendo enterovirus el agente más identificado. La detección de $\mathrm{HPeV}$ aumentó el diagnóstico etiológico viral globalmente a $27 / 59(45,8 \%)$, es decir $17 \%$ (p: 0,086).

En forma diferenciada por los grupos estudiados, el incremento en diagnóstico virológico fue de $8,7 \%$ para los pacientes con muestras de colección, desde $28,2 \%$ $(13 / 46)$ a $36,9 \%,(17 / 46)$, sin co-infecciones. Para el 
grupo de pacientes hospitalizados con síntomas y signos de sepsis y/o meningoencefalitis el aumento en etiología viral fue de $46,2 \%$, desde $30,7 \%$ (4/13) a 76,9\% (10/13) con una co-infección de $\mathrm{HPeV}$ con $\mathrm{EV}$.

La distribución de pacientes se esquematiza en Figura 2.

Descripción de los pacientes con infección por HPeV (n: 11). El promedio de edad fue de 49 días (rango: 18 días-6 meses), con una alta proporción de niños bajo 3 meses de edad, 91\% (10/11), y con predominio de sexo masculino, 63,6\% (7/11).

Los casos se presentaron más frecuentemente en primavera 54,5\% (6/11) y verano $27,3 \%(3 / 11)$.

El 91\% (10/11) se encontraba hospitalizado y $54,5 \%$ $(6 / 11)$ de ellos ingresaron a la UPCP.

Descripción de casos clínicos con observación hospitalaria (n: 9). Las variables clínicas y de laboratorio de estos pacientes se describen en Tablas 1 y 2 .

Los pacientes-casos 1 y 3 fueron reclutados retrospectivamente, y se accedió a su ficha con dispensa de consentimiento informado por el Comité de Ética.

La historia clínica previa al ingreso hospitalario en todos los pacientes se caracterizó por síndrome febril de hasta $48 \mathrm{~h}$ de evolución, rechazo alimentario e irritabilidad. En la Unidad de Urgencia, 6 de 9 pacientes $(66,6 \%)$ presentaron aspecto tóxico, caracterizado por mala perfusión, requirieron reanimación con volumen y fueron hospitalizados en la UPCP por su gravedad.

En el laboratorio destacó leucopenia $(<5.000$ céls/ $\left.\mathrm{mm}^{3}\right)$ en dos tercios de los pacientes y linfopenia $(<2.000$ céls $/ \mathrm{mm}^{3}$ ) en $90 \%$ de ellos. Otros hallazgos a destacar fueron proteína $\mathrm{C}$ reactiva baja, citoquímico de LCR normal y aumento de transaminasas.

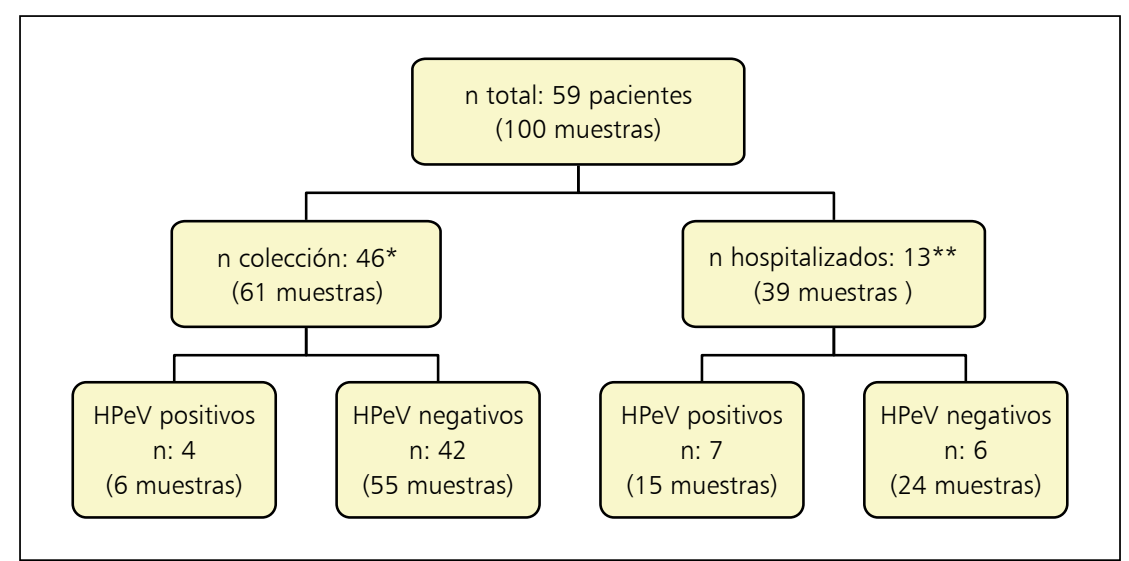

Figura 2. Distribución de pacientes. *13/46 otra etiología: EV, rinovirus, ADV, coronavirus, VHS, influenza, CMV. ${ }^{* *} 4 / 13$ otra etiología: HHV6, rinovirus/coronavirus, norovirus, EV (co-infección HPeV).

A todos los pacientes se les solicitó hemocultivo, urocultivo y cultivo de LCR, todos los cuales fueron negativos a excepción del paciente-caso 3 quien presentó urocultivo positivo para Echerichia coli (Tabla 2).

Dado la similitud clínica entre infección de EV y $\mathrm{HPeV}$, se realizó RPC de EV en fluido estéril (sangre y/o LCR) a todos los pacientes, a excepción del pacientecaso 7 donde se realizó sólo en deposiciones por ser la única muestra disponible. El paciente-caso 2 presentó co-infección con EV en LCR (Tabla 2).

Respecto a la evolución clínica, la fiebre duró en promedio 2,5 días (rango, 1,5-5). El 77\% (7/9) presentó diarrea auto-limitada. Cuatro pacientes presentaron exantema descrito como eritema y edema palmo-plantar (pacientes-casos 4,8 y 9) y pápulas eritematosas en el tronco (paciente-caso 7), con una duración de 2 a 4 días (Figuras 3-5). Todos los pacientes, ante la sospecha de

\begin{tabular}{|c|c|c|c|c|c|c|c|c|c|c|}
\hline & Caso $1 *$ & Caso 2 & Caso $3^{*}$ & Caso 4 & Caso 5 & Caso 6 & Caso 7 & Caso 8 & Caso 9 & Promedio (rango) \\
\hline Edad (días) & 23 & 25 & 19 & 26 & 45 & 68 & 180 & 28 & 30 & $49(19-180)$ \\
\hline Sexo & M & $\mathrm{F}$ & M & M & $\mathrm{F}$ & M & M & M & $\mathrm{F}$ & \\
\hline Fiebre total (h) & 48 & 42 & 48 & 35 & 72 & 120 & 40 & 63 & 25 & $54,7(25-120)$ \\
\hline $\mathrm{T}^{\circ}$ axilar $\left({ }^{\circ} \mathrm{C}\right)$ & 38,2 & 38,8 & 38,5 & 38,7 & 38,6 & 38,6 & 38 & 38,4 & 38,4 & $38,5(38-38,8)$ \\
\hline Tóxico & Sí & Sí & No & Sí & No & Sí & Sí & Sí & No & \\
\hline Exantema & No & No & No & Sí & No & No & Sí & Sí & Sí & \\
\hline Antimicrobianos (días) & 7 & 3 & 14 & 4 & 2 & 10 & 6 & 7 & 2 & $6,1(2-14)$ \\
\hline Estadía UCI/Total (días) & $7 / 7$ & $3 / 4$ & $0 / 5$ & $1 / 5$ & $0 / 6$ & $7 / 11$ & $2 / 6$ & $2 / 5$ & $0 / 3$ & $2,5(0-7) / 6(3-11)$ \\
\hline Mortalidad & No & No & No & No & No & No & No & No & No & \\
\hline
\end{tabular}




\begin{tabular}{|c|c|c|c|c|c|c|c|c|c|c|}
\hline & Caso $1^{*}$ & Caso 2 & Caso $3^{*}$ & Caso 4 & Caso 5 & Caso 6 & Caso 7 & Caso 8 & Caso 9 & Promedio (rango) \\
\hline $\mathrm{PCR}(\mathrm{mg} / \mathrm{dL})$ & 0,13 & 0,36 & 0,65 & 0,55 & 0,07 & 0,21 & 6,6 & 0,72 & 0,06 & $1,04(0,06-6,6)$ \\
\hline Leucocitos (céls./mm³) & 6,4 & 2,9 & 8,7 & 3,9 & 5,9 & 4,19 & 4,12 & 3,3 & 4,8 & $4,9(2,9-8,7)$ \\
\hline Seg/bacil (\%) & $77,8 / 0$ & $52 / 5$ & $48,2 / 0$ & $35,9 / 0$ & $21,3 / 0$ & $24,3 / 0$ & $56 / 0$ & $46 / 7$ & $62 / 0$ & $47(21,3-77,8) / 1(0-7)$ \\
\hline RAN/RAL & $4,9 / 0,5$ & $1,7 / 0,9$ & $4,2 / 3,7$ & $1,4 / 1,8$ & $2,3 / 1,2$ & $1,0 / 1,1$ & $2,3 / 1,5$ & $1,5 / 1,2$ & $3,0 / 0,9$ & $2,5(1-4,9) / 1,4(0,5-3,7)$ \\
\hline Plaquetas (céls/mm³) & 150 & 186 & 403 & 166 & 242 & 159 & 187 & 346 & 233 & $230(150-403)$ \\
\hline \multicolumn{11}{|l|}{ LCR } \\
\hline Glucosa (mg/dL) & 60 & 46 & 85 & 52 & 56 & 61 & 74 & 53 & & $61(46-85)$ \\
\hline Proteínas (mg/dL) & 48 & 57 & 46 & 60 & 22 & 72 & 21 & 35 & & $45(21-60)$ \\
\hline Leucocitos x campo & 0 & 8 & 5 & 3 & 0 & 10 & 0 & 0 & & $3(0-10)$ \\
\hline Gram directo & SB & SB & SB & SB & SB & SB & SB & SB & & \\
\hline Cultivos & Neg & Neg & URC + & Neg & Neg & Neg & Neg & Neg & Neg & \\
\hline Test virales & Neg & $E V+L C R$ & Neg & Neg & Neg & Neg & Neg & Neg & Neg & \\
\hline SGOT (U/L) & & 36 & 31 & 202 & 796 & 564 & 41 & 1376 & 142 & $398(31-1.396)$ \\
\hline SGPT (U/L) & & 34 & 26 & 48 & 180 & 127 & 19 & 214 & 49 & $87(26-214)$ \\
\hline $\operatorname{HPeV}(+)$ & $\begin{array}{c}\text { Plasma } \\
\text { Depo } \\
\text { HNF }\end{array}$ & $\begin{array}{l}\text { LCR } \\
\text { HNF }\end{array}$ & Depo & $\begin{array}{c}\text { Plasma } \\
\text { HNF }\end{array}$ & $\begin{array}{c}\text { Plasma } \\
\text { Depo } \\
\text { HNF }\end{array}$ & Depo & Depo & $\begin{array}{c}\text { Plasma } \\
\text { Depo } \\
\text { HNF }\end{array}$ & $\begin{array}{c}\text { Plasma } \\
\text { Depo } \\
\text { HNF }\end{array}$ & \\
\hline $\operatorname{HPeV}(-)$ & LCR & $\begin{array}{c}\text { Plasma } \\
\text { Depo }\end{array}$ & LCR & $\begin{array}{c}\text { LCR } \\
\text { Depo }\end{array}$ & LCR & $\begin{array}{c}\text { LCR } \\
\text { Plasma }\end{array}$ & & LCR & & \\
\hline Genotipo HPeV & ND & 3 & 3 & 3 & 3 & ND & ND & ND & ND & \\
\hline
\end{tabular}
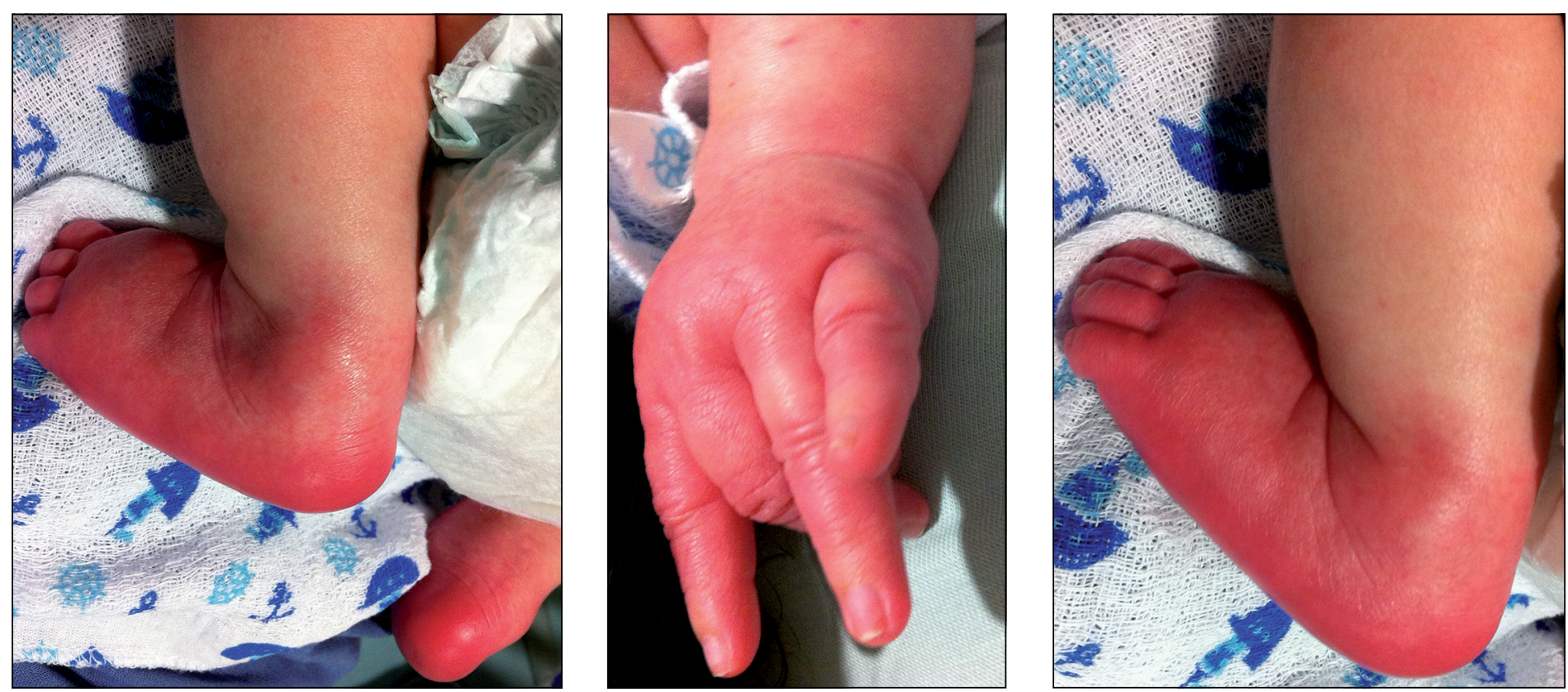

Figuras 3, 4 y 5. Exantema en guante y calcetín de un recién nacido con infección por Parechovirus. 
infección bacteriana grave, recibieron antimicrobianos durante un promedio de 6,1 días (rango 2-14). Sin embargo, sólo en un paciente se confirmó una infección del tracto urinario por E. coli (paciente-caso 3).

El paciente-caso 4 ingresó a pabellón por sospecha de abdomen agudo, sin hallazgos quirúrgicos en la laparotomía exploradora. El paciente-caso 6 presentó crisis convulsiva de difícil manejo durante su hospitalización, evolucionó con hipertonía y requirió apoyo ventilatorio durante cuatro días con cánula de alto flujo, (modalidad de ventilación mecánica no invasora de menor complejidad). Ningún paciente requirió ventilación mecánica invasora ni fármacos vasoactivos durante la estadía en UPCP.

No hubo letalidad asociada entre los casos descritos.

\section{Comparación de los casos positivos y negativos para HPeV}

Dado que se estudiaron dos grupos con características de origen diferentes y el total de casos fue pequeño, no se pudo realizar una comparación exhaustiva entre casos $\mathrm{HPeV}$ positivos y negativos. Sin embargo, al comparar algunas variables se pudo establecer que en el primer grupo de pacientes (muestras de colección), aquellos con $\mathrm{HPeV}$ eran de menor edad, promedio 32 días (18-53) vs 128 días (1-705), sin diferencia en sexo ni procedencia.

En la comparación de los casos hospitalizados, los pacientes con infección por HPeV tenían en promedio dos meses de vida, permanecieron menos días internados y en promedio usaron antimicrobianos dos días menos que los pacientes $\sin \mathrm{HPeV}$. Ninguna de las variables comparadas fue significativa (Tabla 3).

\section{Discusión}

El diagnóstico de sepsis o meningoencefalitis viral en lactantes es difícil, requiere de alta sospecha diagnóstica y de conductas resolutivas rápidas que incluyen una evaluación completa de etiologías bacterianas y virales.

Los síntomas de presentación pueden ser variados; una fracción de los niños se hospitaliza para estudio y manejo y hasta $30 \%$ de ellos puede necesitar asistencia en una UPCP ${ }^{27}$. Las opciones de diagnóstico son variadas, dependiendo de la edad y las manifestaciones clínicas. Entre las etiologías virales, la infección por herpes simplex suele tener un pronóstico ominoso, especialmente en el grupo de recién nacidos que se pueden ver afectados por una infección herpética sistémica, con o sin compromiso del SNC. Un diagnóstico y tratamiento precoces pueden incidir dramáticamente en su posterior morbilidad y letalidad; de hecho, después de la introducción del uso de altas dosis de aciclovir en el tratamiento, $83 \%$ de los recién nacidos con enfermedad diseminada y $31 \%$ de los con compromiso del SNC tienen un desarrollo normal al cumplir un año de vida ${ }^{30}$.

\begin{tabular}{|c|c|c|c|}
\hline & $\begin{array}{l}\mathrm{HPeV}(+) \\
\mathrm{n}: 7\end{array}$ & $\begin{array}{l}\mathrm{HPeV}(-) \\
\mathrm{n}: 6\end{array}$ & p \\
\hline $\begin{array}{l}\text { Edad, días } \\
\text { Promedio (rango) }\end{array}$ & $58(24-187)$ & $138(6-612)$ & 0,187 \\
\hline $\begin{array}{c}\text { Sexo, } n,(\%) \\
\text { Masculino } \\
\text { Femenino }\end{array}$ & $\begin{array}{l}4(57) \\
3(43)\end{array}$ & $\begin{array}{l}3(50) \\
3(50)\end{array}$ & 0,797 \\
\hline Ingreso a UPCP, n, (\%) & $4(57)$ & $5(83)$ & 0,725 \\
\hline $\begin{array}{l}\text { Hospitalización, días } \\
\text { Promedio (rango) }\end{array}$ & $5,7(3-11)$ & $7,6(2-21)$ & 0,488 \\
\hline $\begin{array}{l}\text { Antimicrobianos, días } \\
\text { Promedio, rango }\end{array}$ & $4,8(2-10)$ & $7,4(2-16)$ & 0,344 \\
\hline Mortalidad, n & 0 & 0 & \\
\hline
\end{tabular}

Los EV no polio, son otro gran grupo de virus que pueden ser causa de una variada gama de síntomas de gravedad variable; entre ellos, los más frecuentemente asociados con sepsis, compromiso neurológico y cardiopulmonar grave son el enterovirus 71 y los virus coxsackie serotipos 1 a 5 .

Junto a la mejoría de los recursos de diagnóstico molecular, parechovirus ha surgido como un nuevo virus responsable de fiebre, exantema, enfermedad tipo sepsis y compromiso del SNC. El grupo más afectado es los lactantes pequeños, quienes entre las manifestaciones clínicas mencionadas pueden tener compromiso hepático y trastornos de coagulación ${ }^{27,31}$.

En este estudio se intentó dimensionar la presencia de este agente en muestras de pacientes que fueron estudiados por un diagnóstico sugerente de sepsis viral o compromiso del SNC (muestras de colección). En una segunda fase del estudio se evaluó a niños hospitalizados para estudio de infección sistémica, donde $\mathrm{HPeV}$ participó del diagnóstico diferencial. Si bien, en globo cuantificamos una positividad de $\mathrm{HPeV}$ de $18,6 \%$, esta cifra es mayor a la informada por trabajos previos de 3 a $8 \%{ }^{15,17,19,27}$. Esta diferencia podría ser explicada por la selección de los participantes, donde se centró el reclutamiento en pacientes hospitalizados, lactantes con diagnóstico clínico de sepsis y/o meningoencefalitis, con otras etiologías virales negativas o pendientes de descartar al momento del ingreso al estudio. Además, debido a los tiempos de ejecución del proyecto, coincidió la incorporación de este grupo de pacientes en primavera y verano, lo que aparentemente pudo aumentar la detección de este agente. Esto hace incrementar en forma importante la estimación 
de hallazgo del virus en términos globales pudiendo ser mejor expresado como un rango entre 8,7 y $53,8 \%$ en los lactantes estudiados.

La frecuencia de $\mathrm{HPeV}$ encontrada y el cese del hallazgo de casos positivos, fue una de las razones para publicar este estudio con una población menor a la calculada inicialmente. La importancia de dar a conocer este virus a nivel nacional, su caracterización clínica y la técnica diagnóstica utilizada, obedece al incentivo de su búsqueda frente a pacientes con cuadro clínico sugerente de infección por $\mathrm{HPeV}$.

Desde el punto de vista epidemiológico, los casos predominaron en primavera, lo que difiere con la literatura científica, donde se describe a fines de verano e inicios de otoñ $\mathrm{o}^{31}$; esto puede estar sesgado, porque los pacientes hospitalizados ingresaron al estudio por un período más corto de tiempo, sin incluir todas las estaciones del año. En este sentido, un sistema de vigilancia activo para estos agentes podría dar mejor información de su estacionalidad.

En tanto, el predominio de sexo masculino y edades menores a tres meses concuerda con datos previamente descritos ${ }^{27}$.

En relación a la presentación clínica, todos los pacientes hospitalizados presentaron un cuadro compatible con infección viral sistémica, llamando la atención la irritabilidad marcada, que en uno de los casos se interpretó como debido a dolor abdominal, siendo tan significativa que tuvo como consecuencia una cirugía laparoscópica. El exantema asociado a edema palmo-plantar presente en un tercio de nuestros casos ha sido descrito como manifestación de $\mathrm{HPeV}$ tipo $3^{16,27,34-36}$.

En los exámenes de laboratorio general destacaron parámetros inflamatorios bajos, leucopenia y aumento de transaminasas, en el contexto de un cuadro viral, lo que ha sido reportado en estudios previos ${ }^{32,35}$. El paciente con $\mathrm{HPeV}$ positivo en $\mathrm{LCR}$, presentó un análisis citoquímico de líquido cefalorraquídeo normal para la edad la cual es una de las diferencias descritas al comparar meningoencefalitis por $\mathrm{HPeV}$ con $\mathrm{EV}^{37,38}$.

En el contexto clínico y de laboratorio presentado existe un patrón de sospecha que debe confirmarse con un diagnóstico virológico específico.

Respecto a las muestras y técnicas diagnósticas a utilizar, De Crom y cols. ${ }^{22}$, demostraron diferente sensibilidad de la RPC-TR de HPeV según tipo de muestra, como fue comentado en la Introducción. En nuestro estudio la muestra que logró mayor detección virológica de $\mathrm{HPeV}$ fue también la muestra de deposiciones. Sin embargo, es importante resaltar que como fluido único para diagnóstico de enfermedad actual es insuficiente y resulta necesaria una muestra de fluido estéril positiva para una documentación definitiva del diagnóstico. Sin embargo, en pacientes con clínica altamente sugerente y en ausencia de muestras de fluido estéril, RPC-TR de $\mathrm{HPeV}$ en deposiciones es de gran utilidad para apoyar la hipótesis diagnóstica en ausencia de otra etiología identificada.

Hubo un paciente en el que se identificó EV y HPeV en LCR por RPC en tiempo real comprobándose coinfección por ambos agentes. La especificidad de los partidores para $\mathrm{HPeV}$ descarta una detección cruzada con la detección de EV.

El genotipo 3 de $\mathrm{HPeV}$ fue el único identificado en nuestro estudio y ha sido asociado enfermedad más grave en lactantes bajo 3 meses $^{24-26}$. Habría una explicación biológica por la cual los distintos HPeV diferirían en su tropismo y en su capacidad de diseminación. Los picornavirus entran a la célula a través de la expresión de una secuencia específica de la proteína VP1. En HPeV 1, el carboxilo terminal de la proteína estructural VP1 contiene una secuencia de arginina-glicina-ácido aspártico que es utilizada por muchos virus para lograr adherirse a las integrinas de la superficie celular. HPeV 1 utiliza principalmente las integrinas avb1, avb3 y avb6 como sus receptores ${ }^{10,19,39}$. Es importante mencionar que $\mathrm{HPeV} 3$ difiere de $\mathrm{HPeV} 1$ y de otros parechovirus no codificando esta secuencia en VP1. Esto sugeriría la utilización de un receptor distinto para entrar a la célula, que conlleva a un tropismo diferente, lo que podría explicar mayor compromiso de SNC en HPeV 3. Westerhuis y cols. ${ }^{40}$, estudiaron cepas de HPeV 1 y HPeV 3 en cultivos de células intestinales, respiratorias y neuronas, aplicando RPC en tiempo real con el objetivo de verificar si la cinética de replicación viral in vitro de $\mathrm{HPeV} 1 \mathrm{y} \mathrm{HPeV}$ 3 se relacionaba con la patogenicidad. No se encontró relación al comparar los síntomas clínicos de $\mathrm{HPeV} 1$ y la replicación in vitro de las cepas $\mathrm{HPeV} 1$. Por el contrario, las cepas HPeV 3 mostraron una replicación más rápida en las células neuronales y hubo relación entre la mayor cinética de replicación in vitro y la neuro-patogenicidad en el paciente. Además $\mathrm{HPeV} 1$ pudo ser neutralizado por su anticuerpo específico y por inmunoglobulina endovenosa, mientras que la mayoría de las cepas $\mathrm{HPeV} 3$ no pudieron ser neutralizadas ${ }^{19,40}$. Estos hallazgos podrían apoyar la gravedad de $\mathrm{HPeV} 3$.

El tratamiento de $\mathrm{HPeV}$ es de soporte, no hay tratamiento antiviral disponible; el uso de inmunoglobulina ha sido informada como de utilidad en reporte de casos $^{41}$.

Una identificación de este virus con un cuadro clínico compatible debe plantear la suspensión de los antimicrobianos utilizados en el manejo inicial de un paciente grave y una estadía hospitalaria más breve.

De acuerdo a nuestros resultados, recomendamos realizar RPC-TR para HPeV en plasma y LCR como fluidos más representativos de compromiso de parénquimas estériles y complementar con muestra de deposiciones, dada su alta sensibilidad, la que sería de especial utilidad 
cuando no fuera posible realizar una punción lumbar o, estos dos fluidos arrojaron resultados negativos en un paciente con un caso clínico sugerente. La RPC-TR descrita por W. Allan Nix y cols. ${ }^{11}$, es capaz de detectar todos los genotipos descritos de $\mathrm{HPeV}$ con una alta sensibilidad y especificidad.

Dentro de las limitaciones del estudio se encuentran las características del origen de los pacientes incluidos; un bajo número de casos prospectivos limita una estimación precisa de una cifra real de incidencia. Sin embargo, este estudio logra la identificación de $\mathrm{HPeV}$ en nuestros pacientes y mejora el diagnóstico virológico en situaciones clínicas de sospecha de compromiso viral tipo sepsis o meningitis en lactantes, desde 28,8 a 45,8\%. Las características clínicas son destacables y aportan información de utilidad para estimular la sospecha clínica de estos casos en este grupo de pacientes. Cabe señalar, que este es el primer estudio en Latinoamérica y nacional de este patógeno emergente.

\section{Conclusiones}

La frecuencia de $\mathrm{HPeV}$ en lactantes con sospecha de sepsis y/o meningitis viral varió entre $8,7 \%$ en muestras de colección y $53,8 \%$ en niños hospitalizados en una temporada de incidencia, aparentemente, frecuente.

Si bien las muestras que certifican diagnóstico son sangre y LCR, la positividad de la muestra de deposiciones, que expresa excreción viral por la ruta entérica, puede ser de apoyo al diagnóstico ante una sospecha clínica bien fundamentada y en ausencia de otras muestras como plasma o LCR positivas al $\mathrm{HPeV}$.

El genotipo identificado fue $\mathrm{HPeV} 3$, compatible con cuadros más graves que requirieron hospitalización. Un estudio más amplio de la epidemiología en consulta ambulatoria es necesario para un completo entendimiento del espectro clínico y epidemiológico de este agente viral.

$\mathrm{HPeV}$ es una etiología que debe ser considerada en la actualidad como parte del estudio virológico de sepsis y/o meningitis viral en lactantes. El recurso de RPCTR debiera ser incorporado en centros de referencia de diagnóstico virológico y la circulación del virus debiera sumarse a los sistemas de vigilancia de agentes virales asociados a infecciones graves de la infancia.

Agradecimientos. Al personal del Laboratorio de Infectología y Virología Molecular Red de Salud UC CHRISTUS, Pontificia Universidad Católica de Chile. A Steven Oberste, Centers for Disease Control and Prevention. A Cecilia Vizcaya y Regina Pérez, Infectólogas Pediatras, y Soledad Urzúa, Neonatóloga, todas pertenecientes a la Pontificia Universidad Católica de Chile, por su ayuda en la captación y observación clínica de los pacientes reclutados.

\section{Resumen}

Introducción: Parechovirus humano (HPeV) pertenece a la familia Picornaviridae; ha sido descrito en sepsis viral y meningoencefalitis en niños de dos años o menos (lactantes). Se conocen 16 genotipos, siendo los más frecuentes HPeV 1, 2 y 3; el más grave es el tipo 3. Objetivos: Estimar la frecuencia de $\mathrm{HPeV}$ como agente causal de meningoencefalitis o sepsis viral en lactantes; caracterizar clínica y molecularmente los $\mathrm{HPeV}$ encontrados. Material y Métodos: Estudio descriptivo. Se utilizaron muestras de LCR, plasma, hisopado nasofaríngeo y/o deposiciones de lactantes con sospecha de sepsis y/o meningoencefalitis viral entre octubre 2013 y marzo 2015. Se estudiaron muestras almacenadas en laboratorio y de pacientes hospitalizados. Como diagnóstico, se realizó RPC-TR en tiempo real para HPeV dirigido a sector 5'UTR. Para la caracterización molecular, se secuenció un sector de 304 $\mathrm{pb}$ en unión VP3/VP1 mediante una RPC-TR anidada. Resultados: Se reclutó un total de 59 pacientes con frecuencia de HPeV de 18,6\% (11/59), correspondientes a $8,7 \%$ (4/46) en muestras de colección y 53,8\% (7/13) en hospitalizados, edad promedio 49 días. En la presentación clínica destacó la irritabilidad, el rechazo alimentario y la fiebre. Seis casos fueron genotipificados, todos correspondieron al tipo $\mathrm{HPeV}$ 3. Conclusiones: $\mathrm{HPeV}$ debe ser considerado como agente causal en sepsis y/o meningoencefalitis en lactantes.

\section{Referencias bibliográficas}

1.- Brockmann P, Ibarra X, Silva I, Hirsch T. Etiología del síndrome febril agudo sin foco en niños bajo 36 meses de edad que consultan a un servicio de urgencia. Rev Chilena Infectol 2007; 24 (1): 33-9.

2.- Wylie K, Mihindukulasuriya K, Sodergren E, Weinstock GM, Storch GA. Sequence analysis of the human virome in febrile and afebrile children. PLoS ONE 2012; 7(6): e27735.

3.- Zingg W, Holmes A, Dettenkofer M, Goetting T, Secci F, ClackL,et al. Hospital organisation, management, and structure for prevention of health-care-associated infection: a systematic review and expert consensus. Lancet Infect Dis 2015; 15 (2): 212-24.

4.- Klevens RM, Edwards JR, Richards CL Jr, Horan TC, Gaynes RP, Pollock DA et al. Estimating health care-associated infections and deaths in U.S. hospitals, 2002. Public Health Rep 2007; 122(2): 160-6.

5.- Colvin J, Muenzer J, Jaffe D, Smason A Deych E, Shannon W, et al. Detection of viruses in young children with fever without an apparent source. Pediatrics 2012; 130(6): e1455-62.

6.- Wigand R, Sabin AB. Properties of ECHO types 22, 23 and 24 viruses. Arch Gesamte Virusforsch 1961; 11: 224-47. 
7.- Ghazi F, Hughes PJ, Hyypia T, Stanway G. Molecular analysis of human parechovirus type 2 (formerly echovirus 23). J Gen Virol 1998; 79 (Pt 11): 2641-50.

8.- Johansson ES, Niklasson B, Tesh RB, Shafren DR, Travassos da Rosa AP, Lindberg AM. Molecular characterization of M1146, an American isolate of Ljungan virus (LV) reveals the presence of a new LV genotype. J Gen Virol 2003; 84: 837-44.

9.- Debiaggi M, Canducci F, Ceresola ER, Clementi M. The role of infections and coinfections with newly identified and emerging respiratory viruses in children. Virol J 2012; 9: 247.

10.- Harvala H, Wolthers K, Simmonds P. Parechoviruses in children: understanding a new infection. Curr Opin Infect Dis 2010; 23: 224-30.

11.- Nix WA, Maher K, Johansson ES, Niklasson B, Lindberg AM, Pallansch MA, et al. Detection of all known parechoviruses by real-time PCR. J Clin Microbiol 2008; 46 (8): 2519-24.

12.- Harvala H, Robertson I, McWilliam Leitch EC, Benschop K, Wolthers KC, Templeton K et al. Epidemiology and clinical associations of human parechovirus respiratory infections. J Clin Microbiol 2008; 46 (10): 3446-53.

13.- Harvala H, Simmonds P. Human parechoviruses: Biology, epidemiology and clinical significance. J Clin Virol 2009; 45 (1): $1-9$.

14.- Tapia G, Cinek O, Witsø E, Kulich M, Rasmussen T, Grinde B, et al. Longitudinal observation of parechovirus in stool samples from Norwegian infants. J Med Virol 2008; 80 (10): 1835-42.

15.- Renaud C, Harrison C. Human parechovirus 3 the most common viral cause of meningoencephalitis in young infants. Infect Dis Clin North Am 2015; 29 (3): 415-28.

16.- Piñeiro L. Vicente D, Montes M, HernándezSorronsoro U, Cilla G. Human parechoviruses in infants with systemic infection. J Med Virol 2010; 82 (10): 1790-6.

17.- Abedi GR, Watson JT, Pham H, Nix WA, Oberste MS, Gerber SI. Enterovirus and human parechovirus surveillance - United States, 2009-2013. MMWR Morb Mortal Wkly Rep 2015; 64 (34): 940-3.

18.- Sharp J, Bell J, Harrison CJ, Nix WA, Oberste MS, Selvarangan R. Human parechovirus in respiratory specimens from children in Kansas City, Missouri. J Clin Microbiol 2012; 50 (12): 4111-3.
19.- Sposito S, Rahamat-Langendoen J, Ascolese B, Senatore L, Castellazzi L, Niesters H G. Pediatric parechovirus infections. J Clin Virol 2014; 60 (2): 84-9.

20.- Hashem M, Menegus M. Back to basics: Molecular tools in the diagnosis and management of infectious diseases. Pediatr Rev 2005; 26 (1): 15-20.

21.- Levorson R, Jantausch B. Human parechovirus. Pediatr Infect Dis J 2009; 28 (9): 831-2.

22.- De Crom S.C.A, Obihara C.C, de Moor R.A, Veldkamp EJ, van Furth AM, Rossen JW. Prospective comparison of the detection rates of human enterovirus and parechovirus RTqPCR and viral culture in different pediatric specimens. J Clin Virol 2013; 58 (2): 449 - 54.

23.- Chuchaona W, Khamrin P, Yodmeeklin A, Saikruang W, Kongsricharoern T, Ukarapol N, et al. Detection and characterization of a novel human parechovirus genotype in Thailand. Infect Genet Evol 2015; 31: 300-4.

24.- Kolehmainen $P$, Jääskeläinen $A$, Blomqvist $S$, Kallio-Kokko H, Nuolivirta K, Helminen M, et al. Human parechovirus type 3 and 4 associated with severe infections in young children. Pediatr Infect Dis J 2014; 33: 1109-3.

25.- Sedmak G, Nix WA, Jentzen J, Haupt T, Davis J, Bhattacharyya S, et al. Deaths associated with human parechovirus infection in Wisconsin. Clin Infect Dis 2010; 50 (3): 357-61.

26.- van Zwol A L, Lequin M, Aarts Tesselaar C, van der Eijk A, A Driessen G, De Hoog M, et al. Fatal neonatal parechovirus encephalitis. Br Med J Case Rep 2009; 2009: bcr05.2009.1883.

27.- Khatami A, McMullan B.J, Webber M, Stewart P, Francis S, Timmers K J, et al. Sepsis-like disease in infants due to human parechovirus type 3 during an outbreak in Australia. Clin Infect Dis 2015; 60 (2): 228-36.

28.- Estadísticas epidemiológicas de código abierto para Salud Pública http://www.openepi. com/Menu/OE_Menu.htm (accedido: $10 \mathrm{de}$ septiembre de 2013).

29.- Harvala H, Robertson I, Chieochansin T, McWilliam Leitch E C, Templeton K, Simmonds P. Specific association of human parechovirus type 3 with sepsis and fever in young infants, as identified by direct typing of cerebrospinal fluid samples. J Infect Dis 2009; 199 (12): 1753- 60.

30.- Kimberlin D W, Lin C Y, Jacobs R F, Powell D A, Corey L, Gruber W C, et al. Safety and efficacy of high-dose intravenous acyclovir in the management of neonatal herpes simplex virus infections. Pediatrics 2001; 108 (2): 230-8.

31.- Levorson R E, Jantausch B A,Wiedermann B L, Spiegel H M, Campos J M. Human parechovirus-3 infection: emerging pathogen in neonatal sepsis. Pediatr Infect Dis J 2009; 28: 545-7.

32.- Renaud C, Kuypers J, Ficken E, Cent A, Corey L, Englund J A. Introduction of a novel parechovirus RT-PCR clinical test in a regional medical center. J Clin Virol 2011: 51 (1): 50-3.

33.- Davis J, Fairley D, Christie S, Coyle P, Tubman R, Shields M D. Human parechovirus infection in neonatal intensive care. Pediatr Infect Dis J 2015; 34: 121-4.

34.- Piralla A, Mariani B, Stronati M, Marone P, Baldanti F. Human enterovirus and parechovirus infections in newborns with sepsis-like illness and neurological disorders. Early Hum Dev 2014; 90 S1: S75-7.

35.- Eyssette-Guerreau S, Boize P, Thibault M, Sarda H. Neonatal parechovirus infection, fever, irritability and myositis. Arch Pediatr 2013; 20 (7): 772-4.

36.- ShojiK, Komuro H, KobayashiY,Shike T, Funaki T, Katsuta T, et al. An infant with human parechovirus type 3 infection with a distinctive rash on the extremities. Pediatr Dermatol 2014; 31 (2): 258-9.

37.- Sharp J, Harrison C, Puckett K, Selvaraju S B, Penaranda S, Nix W A, et al. Characteristics of young infants in whom human parechovirus, enterovirus or neither were detected in cerebrospinal fluid during sepsis evaluations. Pediatr Infect Dis J 2013; 32: 213-6.

38.- Cabrerizo M, Trallero G, Pena M J, Cilla A, Megias G, Muñoz-Almagro C, et al. Comparison of epidemiology and clinical characteristics of infections by human parechovirus vs. those by enterovirus during the first month of life. Eur J Pediatr 2015; 174 (11): 1511-6.

39.- Joki-Korpela P, Marjomaki V, Krogerus C, Heino J, Hyypiä T. Entry of human parechovirus 1. J Virol 2001; 75 (4): 1958-67.

40.- Westerhuis B M, Koen G, Wildenbeest J G, Pajkrt D, de Jong M D, Benschop K S, et al. Specific cell tropism and neutralization of human parechovirus types 1 and 3: implications for pathogenesis and therapy development. J Gen Virol 2012; 93 (Pt 11): 2363-70.

41.- Wildenbeest J G, Wolthers K C, Straver B, Pajkrt D. Successful IVIG treatment of human parechovirus-associated dilated cardiomyopathy in an infant. Pediatrics 2013; 132 (1): e243-7. 